\title{
Logical reasoning, world knowledge, and mental imagery: Interconnections in cognitive processes
}

\author{
CATHERINE A. CLEMENT and RACHEL JOFFE FALMAGNE \\ Clark University, Worcester, Massachusetts
}

\begin{abstract}
To better understand the role of problem content in verbal reasoning, the effect of two aspects of problem representation on conditional reasoning was examined. Specifically, this study focused on the effect of availability of knowledge schemata and mental imagery on recognition of indeterminacy. Four groups of 20 adults solved syllogisms that varied in imagery value and in tendency to access knowledge schemata (assessed by ratings of the relatedness of antecedent and consequent clauses of premises). When problems both were high in imagery value and had related clauses, performance was significantly better on indeterminate syllogisms. Access to schemata may permit elaborative processing and the generation of counterexamples to invalid inferences; imagery may support representation of problems and generation of elaborative information in memory.
\end{abstract}

It has been well documented that meaningful thematic material can influence the reasoning process in deductive reasoning tasks, most notably by improving subjects' differentiation between logically necessary and logically indeterminate inferences (e.g., Kuhn, 1977; Wason \& Johnson-Laird, 1972). However, the particular properties of this material that are effective and the reasons why they are effective have not been characterized in specific terms. Understanding this phenomenon in more detail requires examining the specific interplay in reasoning tasks between knowledge of logical relations and knowledge related to problem content, and the relationship between the mental representation of a problem and the reasoning process.

Given that normal sentence-comprehension processes are likely to be involved in processing meaningful verbal reasoning problems, research in text comprehension suggests two specific ways in which particular problem contents may affect a problem's mental representation. First, when a problem is formulated with thematic content, elaborative processing of the problem can occur through accessing knowledge schemata in memory. Second, certain features of meaningful content may elicit an imaginal representation of the problem. The present article addresses the effect of these two aspects of problem representation on reasoning processes in solving conditional syllogisms, particularly on handling indeterminate problem forms.

This paper is based on a master's thesis submitted to the Department of Psychology, Clark University. A version of the paper was presented at the meetings of the Eastern Psychological Association, April 1983, Philadelphia, PA. We wish to thank Robert Mitchell and Ronald Mawby for helpful comments on an earlier draft.

Requests for reprints should be sent to Catherine Clement, Department of Psychology, Clark University, 950 Main St., Worcester, MA 01610.
The experiments reported in this article focus on conditional reasoning in standard conditional reasoning tasks, that is, evaluating implications of statements formed with the conditional connective "if $p$ then $q$." There are four basic syllogistic forms associated with the connective; two yield determinate solutions and two are indeterminate. ${ }^{1}$ To distinguish determinate from indeterminate problems, subjects must realize that the conditional relation between connected propositions $(p, q)$ is asymmetrical; the antecedent implies the consequent, but the converse is not necessarily the case. Overlooking this constraint leads subjects to invalidly conclude from the premise "if $p$ then $q$ " that "if $q$ then $p$," that is, treating the connective as biconditional (and therefore all the syllogisms as determinate).

Often in conditional reasoning tasks, subjects correctly reason given determinate problems (especially modus ponens) but treat indeterminate problems as determinate. For example, when told "if $p$ then $q$ " and "not $p$," they often do not recognize that no definite conclusion can then be reached about $q$.

Accounts given for this finding suggest that, in natural language, the "if...then" connective is ambiguous (Wason \& Johnson-Laird, 1972) or vague (Braine, 1977; Braine, Reiser, \& Rumain, 1984) and that discourse conventions often lead individuals to assume that the "invited" inference "if $q$ then $p$ " is valid. However, the semantics of sentence content can affect inferences drawn, for example, by constraining the initial interpretation of a conditional premise as either conditional or biconditional (Revlin \& Leirer, 1978; Staudenmayer, 1975), or by suggesting counterexamples to invalid inferences (a state of affairs in which the antecedent is false and the consequent true; Kuhn, 1977; Wason \& Johnson-Laird, 1972).

In the work just cited, content effects have been discussed in general terms only, with reference to such global constructs as meaningfulness or familiarity. One aim of 
the present research is to examine content effects more specifically, in terms of variables that are theoretically meaningful given research on sentence and text processing.

It is widely accepted (e.g., Anderson \& Reder, 1979; Bransford \& Franks, 1971; Reder, 1980; Rumelhart, 1980; Schank, 1981) that text comprehension entails the elaboration of incoming information with prior knowledge structures (variously characterized as scripts, schemata, or elaborative productions). These structures consist of information organized around different classes of events, specifying both invariant and optional aspects of these events. Accessing these schemata aids interpretation of text by permitting inferences concerning what is necessarily or plausibly true of a situation described.

In this study, we examined the role of such elaborative processing in conditional reasoning tasks. A specific property of meaningful material that may affect schema accessibility was identified: the "relatedness" of clauses of conditional statements. Clauses may be related in a readily apparent or natural way, or their relation can be odd or unusual but still meaningful. For example, in the sentence "If the man wants plain doughnuts, then he walks to the corner bakery," existing schemata are readily accessed in which these two events are related (note, however, there is no necessary relation involved). In contrast, in the sentence "If the man walks his dog, then he has fish for breakfast," the clauses do not seem naturally related; an existing knowledge structure is less likely to be accessed by this sentence, although the sentence is meaningful.

If a situation described in a conditional reasoning problem accesses an existing knowledge structure, then additional schema-related knowledge may be available for elaboration of the problem. Such knowledge may be important to the recognition of indeterminacy if it allows the generation of imagined states of affairs in which the consequent occurs with alternative antecedents (these can provide counterexamples to invalid inferences). On the other hand, if the antecedent and consequent are not easily incorporated into an existing knowledge structure, little schema-related information can be used for elaboration, even though the sentence is still meaningful. Also, individuals may then ignore content and treat the problem formally, relying exclusively on explicitly represented knowledge, which may be inaccurate, of the "if. ..then" connective. Thus the relatedness of clauses, in the sense discussed above, was investigated here in order to understand the role of the availability of knowledge schemata in the reasoning process. Schema accessibility per se, as distinct from meaningfulness, has not been manipulated in previous studies.

It is important to note that the content of problems used in this study was neutral and not empirically biased. Premises of problems described invented situations which, if real, could be either biconditional or conditional (relations between clauses were arbitrary), and conclusions to problems could be either true or false. Thus, counter- examples to invalid inferences could not be generated from factual knowledge or episodic memories. Rather, they would be generated from knowledge of possible values of variables in a general schema, that is, from knowledge of what kinds of events are possible within that schema, (e.g., given a bakery and pastry schema, a subject could generate ideas about a man going to the corner bakery for cookies instead of doughnuts).

Our assumption that generalized knowledge can influence the reasoning process contrasts with recent proposals by Cox and Griggs (1982) and Pollard (1982) that content effects are only found when factual knowledge and episodic memories are available for application to reasoning tasks. These investigators also propose that such available knowledge provides the sole basis for conclusions drawn at the exclusion of logical processes. Difficulties with both of these proposals will be considered in the final discussion.

A second aspect of content, identified in research on text comprehension, that can affect the processing of verbal material concerns the formation of mental imagery. A number of studies indicate that people perform verbal tasks differently depending on the availability of imagery (e.g., see Eddy \& Glass, 1981; Kosslyn, 1980, 1981; Marschark \& Paivio, 1977; Paivio, 1971; Shaver, Pierson \& Lang, 1974). Whatever the ultimate nature of imaginal representations (for alternative views, see Kosslyn, 1980, 1981; Pylyshyn, 1981), images do appear to play a distinct functional role in cognition (see Block, 1981; Fodor, 1981; Kaufmann, 1980; Shepard, 1978).

The only direct examination of imagery in verbal conditional reasoning has been by Falmagne, Singer, and Clement (1985). Adults were given problems, all of which were meaningful, but which varied in terms of imagery value. Performance on indeterminate syllogisms was significantly better among subjects reasoning with highimagery material, although performance on determinate problems was relatively unaffected. This interaction of imagery with logical form indicated that imagery functioned at the level of logical processing and not simply in sentence comprehension. Following Falmagne (1980), Falmagne et al. proposed that a well mastered, formal mode of representation and processing was unavailable for the more difficult indeterminate syllogisms. Therefore, for these problems, subjects given high-imagery material putatively used an imaginal medium to represent information in working memory. This imaginal representation served as input for subsequent logical processing. In contrast, for the easier, better mastered determinate problems, a formal mode of representation could be used.

The purpose of the present study was to elucidate the role of accessibility to knowledge schemata, and the way in which this articulates with mental imagery, in influencing recognition of indeterminacy in conditional reasoning tasks. Given the preceding analysis, clause relatedness should allow access to knowledge schemata and elaborative processing and thus permit the generation of counterexamples to invalid inferences. Imagery, as 
Falmagne et al. (1985) suggest, may provide an alternative mode of representation of the difficult, indeterminate problems and thus support logical processing in working memory. Although that study provided evidence for the importance of imagery, it did not examine its interplay with schema accessibility. One of the aims of the present study was to investigate the specific way in which these two processes may articulate with one another especially regarding elaborative processing. Furthermore, the analysis outlined in this article suggests that there was a possible confound in the earlier study, revealed by examination of material used. Conditional sentences with high-imagery material may have more easily led to the access of knowledge schemata because of the relatedness of clauses. Low-imagery sentences seemed to have relatively unrelated clauses. Thus, an additional aim of the present study was to provide clarification of the previous results.

Experiment 1 examined performance on conditional reasoning problems differing in "clause relatedness" and, orthogonally, in imagery value. If imagery value is a primary factor in facilitating recognition of indeterminacy, then it should improve performance regardless of the level of clause relatedness. If, on the other hand, access to knowledge schemata is the main factor, and clause relatedness enables such access to occur, then clause relatedness should enhance performance across levels of imagery value. Finally, if the two variables work in conjunction in producing content effects, then the form of the interaction should reveal the particular way in which they articulate.

The above predictions for content effects specifically apply to indeterminate problems. Determinate syllogisms do not differentiate between a conditional or biconditional interpretation of a problem. Thus subjects' recognition of the asymmetry would not be apparent from responses to determinate syllogisms. However, observation of these responses does permit us to distinguish content effects that are specifically related to recognition of indeterminacy from content effects that are related to other aspects of processing conditional statements.

\section{EXPERIMENT 1}

\section{Method}

\section{Subjects}

Subjects were Clark University undergraduate students and staff members, all volunteers. Preliminary rating tasks required 90 subjects; 80 other subjects participated in the conditional reasoning task.

\section{Materials and Design}

Rating tasks. In order to develop problems that varied in terms of imagery value and clause relatedness, two types of rating tasks were administered. In an imagery rating task, subjects rated simple sentences, which could be used subsequently as clauses of conditional statements, on a scale of 1 to 5, according to the ease with which they formed mental pictures of the sentence content. Instructions emphasized that subjects rate according to their spontaneous formation of a mental image-subjects were told to consider how easily and quickly the sentence arouses a mental image. Two sets of 48 simple sentences were constructed.

In a relatedness rating task, subjects rated conditional statements according to how easily or naturally they could conceive of a relation between the two actions described by the constituent clauses. They rated sentences on a scale of 1 to 5 in terms of whether a relation between the two actions seemed readily apparent or not apparent. Instructions stressed that subjects consider their spontaneous reaction. Subjects were told not to seek a necessary relation, but rather a natural one. Finally, they were asked to give a rating of " 0 " if a connection between the two clauses seemed impossible. Sentences rated as impossible were to be eliminated: "unrelated clause" sentences were intended to be those for which subjects had difficulty identifying a relation between clauses, not those for which a relation is believed to violate real-world facts.

Two sets of approximately 40 conditional sentences were rated for relatedness. Approximately half were composed from simple sentences rated high and half from those rated low in the imagery rating task.

Pretest. A logical reasoning pretest was administered in order to match subjects across groups. The test consisted of 18 determinate and indeterminate quantified syllogisms and three-term series problems. $^{2}$

Conditional reasoning task. Conditional reasoning problems varied in terms of imagery value and in terms of clause relatedness. Each content factor had two levels; those materials receiving the highest and lowest mean ratings (and relatively low standard deviations) for each content dimension were selected for construction of problems.

The two content factors were crossed to yield four conditions varied among groups of 20 subjects each. Thus, different groups received problems in which clauses of conditional statements were either (1) high in imagery value and related (HR group); (2) high in imagery value and unrelated (HU group); (3) low in imagery value and related (LR); or (4) low in imagery value and unrelated (LU). Sentences used for problems were selected so that problems in each contrast group had comparable ratings along the dimension that was not being contrasted.

Conditional reasoning tasks within each content group included problems formulated from six specific sentence contents. Each condition contained sentences comparable in terms of length and Thorndike-Lorge frequency ratings of constituent words. The clauses of each statement described an action of a single agent and were stated in the present tense. Attempts were made to use content that was neutral and not empirically biased. That is, content was intended not to describe situations likely to be (1) true or false in the real world, (2) necessarily conditional, or (3) probably biconditional in the real world.

An example sentence from each condition follows: (1) highimagery related clause (HR)-If the man wants plain doughnuts, then he walks to the bakery across the intersection; (2) high-imagery unrelated clause (HU) - If the man walks his golden retriever, then he gets upset about his insect bite; (3) low-imagery related clause (LR)-If the woman reorganizes the company structure, then she makes a profit for the year; and (4) low-imagery unrelated clause (LU)-If the man takes an economic perspective, then he uses the new memory technique.

For the six sentences in each content condition, mean relatedness ratings, imagery ratings, and sentence length, respectively, were: $\mathrm{HR}-4.3,4.3,15$ words; $\mathrm{HU}-2.0,4.3,14.5$ words; $\mathrm{LR}-$ $4.3,2.4,14.5$ words; $L U-2.2,1.9,13.7$ words.

Subjects in each content group were given a conditional reasoning task consisting of 18 syllogisms. There were 3 syllogisms of each of the four logical forms, modus ponens (MP), modus tollens (MT), denying the antecedent (DA), and affirming the consequent (AC). Additionally, there were three modus ponens problems with 
a negative in the consequent of the first premise (MPN; e.g., "If the man wants plain doughnuts, then he does not walk to the bakery at the intersection"), and three modus tollens with a negative in both the antecedent and consequent (MTN). These were included in order to balance the number of "yes," "no," and "can't tell" correct responses. (MP, MT, MPN, and MTN are determinate syllogisms, AC and DA are indeterminate).

Each of the six specific sentence contents in a condition was matched once with each logical form. Thus 36 problems within each content condition represented all possible pairings of specific content and logical form. However, individual subjects received only 18 problems. Two subgroups were formed of half the subjects in each content group, and two sentence subsets of half the specific sentence contents were formed. The pairing of sentence subset with logical form was counterbalanced across subgroups. Task length was limited in this way in order to reduce subject fatigue and repeated exposure to a particular content.

The conditional reasoning task was divided into three blocks, each of which included one problem of each logical type and each specific content. Order of presentation of problems within blocks was constant across subjects and was random, with the restriction that no two problems with the same content occur consecutively between blocks. Furthermore, the final problem in each block was either an AC, DA, or MT inference. Written response justifications, which were of interest only for these logical forms, were requested for the final problem in each block. (These justifications proved difficult to classify and will not be included in the presentation of results.) Order of presentation of the three blocks was counterbalanced across subjects.

\section{Procedure}

Rating tasks. The imagery and relatedness rating tasks were each given to four groups of subjects (average $n=13$ ). For each rating task, two sets of sentences were each read aloud in alternate orders to two groups.

Conditional reasoning task. Conditional reasoning problems were administered to small groups of 1 to 4 subjects. Problems were read aloud. Subjects were asked to listen to two sentences and a question and to give an answer based only on the information provided by the sentences. Subjects were to answer either "yes," meaning "definitely yes," "no," meaning "definitely no," or "can't tell," meaning the sentences did not give enough information to permit a definite answer. Examples of class syllogisms were read and answered.

A randomly chosen subset of individuals ( 8 or 9 subjects) in each content group was given an interview immediately after the main task. Interviewed subjects were asked to give response justifications to problems that were re-presented to them during the interview. Additionally, subjects were asked whether or not they typically formed visual images during the main task; if subjects claimed to visualize, they were asked to describe their images to specific problems presented again.

\section{Results}

Analyses concerned (1) the relative success of each group on indeterminate and determinate problems, (2) imagery reports, and (3) response justifications.

The percentages of correct answers to indeterminate syllogisms in each content group are shown in Table 1. A $2 \times 2 \times 2$ (imagery value $\times$ clause relatedness $\times$ logical form-AC vs. DA) ${ }^{3}$ ANOVA yielded no significant main effects. However, a significant interaction was found between imagery value and clause relatedness $[F(1,76)=$ $5.49, p<.05]$. ANOVAs on the simple main effects of the imagery $\times$ relatedness interaction revealed that the
HR group performed significantly better than the LR group $[F(1,38)=6.45, p=.015]$ and also significantly better than the $\mathrm{HU}$ group $[F(1,38)=7.31, p<.01]$. No other simple main effects (LR vs. LU; HU vs. LU) were significant.

Paradoxically, the HR group was not statistically different from the LU group, although a significant interaction with logical form accounts for this result $[F(1,38)=8.81$, $p<.01]$. The HR group exceeded the LU group on the DA but not the AC inference. An interaction with logical form was also found when the HU and LU groups were compared $[F(1,38)=7.7, p<.01]$, again due to the relatively high performance of the LU group on AC only. This inconsistent performance of the LU group also accounts for an overall interaction between imagery value and logical form found in the original three-way ANOVA $[F(1,76)=7.46, p<.01]$. This irregularity will be considered in the final discussion.

Thus, for indeterminate syllogisms, analyses of the imagery $\times$ relatedness interaction suggest that when high imagery and clause relatedness were both present, significantly more "can't tell" responses were given to indeterminate problems. In contrast, when imagery value was low, clause relatedness did not improve performance, and when clauses were unrelated, high imagery value was not effective.

The analyses of determinate logical forms revealed a different pattern of results. The percentages of correct responses in the various content conditions are shown in Table 2 for syllogisms with affirmative premises and for syllogisms containing negations in the conditional premise. For affirmative syllogisms, the main effect of

Table 1

Percentage of Correct Answers to Indeterminate Problems for Each Experimental Group

\begin{tabular}{cccc}
\hline Group* & $\begin{array}{c}\text { Affirming } \\
\text { the Consequent }\end{array}$ & $\begin{array}{c}\text { Denying } \\
\text { the Antecedent }\end{array}$ & $\begin{array}{c}\text { Total } \\
\text { Indeterminates }\end{array}$ \\
\hline HR & 55 & 68 & 62 \\
HU & 27 & 40 & 34 \\
LR & 37 & 38 & 38 \\
LU & 50 & 37 & 44 \\
\hline
\end{tabular}

Note-HR = high imagery value, related clause material; $\mathrm{HU}=$ high imagery value, unrelated clause material; $L R=$ low imagery value, related clause material; $L U=$ low imagery value, unrelated clause material. ${ }^{*} n=20$ for each group.

Table 2

Percentage of Correct Answers to Determinate Problems for Each Experimental Group

\begin{tabular}{|c|c|c|c|c|}
\hline \multirow[b]{2}{*}{ Group* } & \multicolumn{2}{|c|}{ Affirmative Premise } & \multicolumn{2}{|c|}{ Negations in Premise } \\
\hline & $\begin{array}{l}\text { Modus } \\
\text { Ponens }\end{array}$ & $\begin{array}{l}\text { Modus } \\
\text { Tollens } \\
\end{array}$ & $\begin{array}{l}\text { Modus } \\
\text { Ponens } \\
\end{array}$ & $\begin{array}{l}\text { Modus } \\
\text { Tollens }\end{array}$ \\
\hline HR & 100 & 75 & 98 & 48 \\
\hline HU & 93 & 80 & 97 & 50 \\
\hline LR & 93 & 72 & 92 & 57 \\
\hline LU & 95 & 58 & 77 & 33 \\
\hline
\end{tabular}

Note-HR $=$ high imagery value, related clause material; $\mathrm{HU}=$ high imagery value, unrelated clause material; $L R=$ low imagery value, related clause material; $L U=$ low imagery value, unrelated clause material. ${ }^{*} n=20$ for each group. 
logical form was significant $[F(1,76)=41.56$, $p<.001]$; as is usually found, modus ponens was easier than modus tollens. The main effect of imagery value was also significant $[F(1,76)=4.12, p<.05]$, but inspection of the specific means reveals that the main effect of imagery is primarily due to the low performance of the LU group on the modus tollens inference.

For determinate syllogisms with negations, the main effect of imagery just missed significance $[F(1,76)=3.78$, $p<.055]$, and the main effects for logical form and relatedness were significant $[F(1,76)=126.27, p<.001$, and $F(1,76)=4.58, p<.05$, respectively]. Also, there was a significant interaction between imagery value and clause relatedness $[F(1,76)=4.54, p<.05]$. Effects found are again a product of poor performance by the LU group. Analysis of the simple main effects showed that the percentage of correct responses was significantly lower in the LU group than in the HU and the LR groups $[F(1,38)=5.97, p<.05$, and $F(1,38)=7.96, p<.01$, respectively].

Thus, for determinate syllogisms, particularly those with negations, it appears that high imagery value and clause relatedness had some influence on performance. The effect was most apparent when these two properties were simultaneousiy absent: performance among LU subjects was particularly low relative to the other groups. Schematically, it appears that for indeterminate problems, both factors must be present for positive effects to obtain. In contrast, for determinate problems, both factors must be absent for negative effects to obtain.

An additional analysis of success by each group was an assessment of the number of subjects in each group whose response patterns were consistently in accord with either a conditional or biconditional logic. Classification of subjects as consistently conditional required that those subjects made correct responses to all problems (excluding determinate problems with negations) on the conditional reasoning task. Classification of subjects as consistently biconditional required that those subjects made correct responses to each affirmative determinate problem, a "yes" to each AC, and a "no" to each DA syllogism. As shown in Table 3, there are consistently conditional subjects only in the HR group. The HR group had no consistently biconditional subjects, whereas there were 8 such subjects in the HU, 4 in the LR, and 1 in

Table 3

Number of Subjects in Each Group Who Gave a Consistently Conditional or Consistently Biconditional Response Pattern

\begin{tabular}{ccc}
\hline Group & Conditional & Biconditional \\
\hline HR & 4 & 0 \\
HU & 0 & 8 \\
LR & 0 & 4 \\
LU & 0 & 1 \\
\hline
\end{tabular}

Note-HR $=$ high imagery value, related clause material; $\mathrm{HU}=$ high imagery value, unrelated clause material; $L R=$ low imagery value, related clause material; $L U=$ low imagery value, unrelated clause material. ${ }^{*}=20$ for each group.
Table 4

Response Justifications of Interviewed Subjects to Indeterminate Problems

\begin{tabular}{ccccc} 
& Specific & Nonspecific & & \\
Group & Counterexamples & Counterexamples & Assymetry & Other \\
\hline HR $(n=8)$ & $6 / 12$ & $6 / 12$ & & \\
HU $(n=9)$ & $1 / 3$ & $1 / 3$ & & $1 / 3$ \\
LR $(n=9)$ & $2 / 5$ & $1 / 5$ & $1 / 5$ & $1 / 5$ \\
LU $(n=8)$ & $1 / 7$ & $3 / 7$ & $1 / 7$ & $2 / 7$ \\
\hline
\end{tabular}

Note-The values represent the frequency of each type of justification among the number of correct responses given to indeterminate problems presented during interviews.

the LU group. These findings are consistent with the first set of analyses (but not trivially implied by them).

During posttask interviews, subjects were asked about their use of imagery during the main task. The percentage of interviewed subjects in each group who reported forming images was higher for the HR than for the other groups. The percentage who reported using imagery consistently was $88 \%$ in the HR group and $44 \%, 33 \%$, and $0 \%$ in the HU, LR, and LU groups, respectively. Of the subjects in each of the HU, LR, and LU groups, $22 \%-25 \%$ reported using imagery part of the time.

Subjects were asked to describe mental images they formed for problems presented during the interview. Sometimes these images represented only the given premise, whereas other times they reflected the transformation of information during the problem-solving process. For example, images reported for indeterminate syllogisms sometimes represented counterexamples to invalid inferences (to the necessary coexistence of $p$ and $q$ ). For example, one subject, given the sentence "If the woman wears brown boots to the party, then she wears her new dress," reported an image of a woman wearing her dress with sandals rather than boots.

Response justifications for $\mathrm{AC}$ and DA syllogisms were also requested during interviews. Three categories of justifications for correct responses were derived: (1) specific counterexamples (e.g., given the statement "If the man wants plain doughnuts, then he walks to the bakery at the intersection," subjects might state, "The man could go to the bakery for a danish"); (2) mention of the possibility of counterexamples (e.g., "The man could have gone to the bakery anyway"); (3) explicit mention of asymmetry (e.g., "The sentence only tells you what happens if the man wants plain doughnuts; it doesn't tell you what happens if he walks to the bakery").

The number of justifications of each kind for problems presented during interviews is shown in Table 4. Comparisons across groups are difficult since few correct responses were given by any but HR subjects. We see that all responses by HR subjects included specific or nonspecific counterexamples. Responses in other groups included mention of counterexamples as well as reference to the asymmetry of conditional statements or other justifications that were too incomplete to classify. The LU group mentioned proportionally fewer specific counterexamples. 
In summary, for indeterminate problems, the HR group gave significantly more correct responses than either the HU or LR groups. Also, only in the HR group were there consistently conditional subjects. Thus, when highimagery value and clause relatedness were both present, reasoning with indeterminate syllogisms improved. In contrast, for determinate syllogisms, particularly those with negations, effects were found only when problems were both low in imagery value and unrelated; performance of LU subjects was impaired.

It appears that clause relatedness and imagery value work together to improve performance on indeterminate syllogisms and that neither factor operating alone is sufficient. However, the contributions of the content manipulations to the present results need clarification. The imagery reports seem to indicate that more images were formed by HR subjects than by HU subjects. This raises the possibility that clause relatedness is necessary for the formation of images of the conditional sentences, and thus that its primary importance may have been to permit imagery rather than access to knowledge schemata; $\mathrm{HU}$ subjects then, in fact, may have received low-imagery material. (Recall that imagery value was originally determined from other subjects' ratings to the simple component clauses included in the conditional statements.) Experiment 2 was conducted in order to examine this question by assessing the imagery value of the conditional sentences used in Experiment 1.

\section{EXPERIMENT 2}

\section{Method}

Subjects

Subjects were 24 Clark University undergraduate students.

Materials and Procedure

An imagery rating task was administered using the same procedure as in Experiment 1. Task materials consisted of the 24 conditional sentences used for the reasoning problems in Experiment 1. Two orders of presentation were read aloud to different groups of subjects.

\section{Results}

Results of the new rating task supported the assessment of sentences in Experiment 1. The mean ratings for each sentence type were: HR 4.6; HU 4.0; LR 1.9, and LU 1.5 (there were six sentences of each type). Figure 1 shows the distributions of individual mean imagery rating scores.

As shown in the figure, high-imagery sentences that are related and unrelated are clearly distinct from the lowimagery sentences in terms of imagery value (HR vs. $L R$, $p<.01$; HU vs. LU, $p<.01$, Wilcoxon signed-ranks test). This figure also shows that related sentences received higher ratings than did corresponding unrelated sentences; this difference is also significant (HR vs. HU, $p<.01$; LR vs. LU, $p<.01$ ). However, Figure 1 clearly indicates that the major difference is between highimagery sentences on the one hand and low-imagery sentences on the other hand.

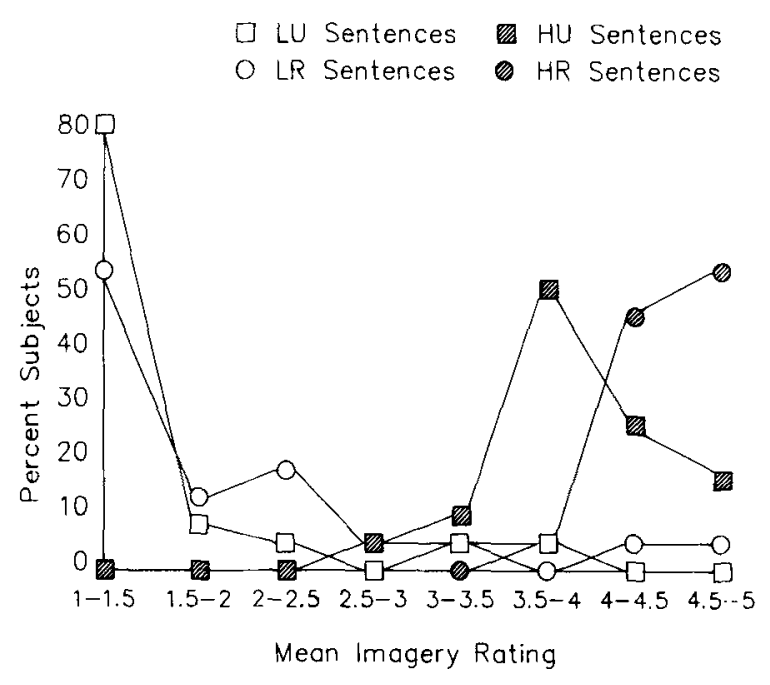

Figure 1. Distribution of individual mean ratings to each type of sentence: HR, HU, LR, and LU.

In relation to results of Experiment 1 regarding indeterminate problems, the present results indicate that the quasi-equivalence of the HU group to the $L R$ and $L U$ groups was not due to an operationally low level of imagery in the HU material. The original explanation of results of Experiment 1 thus remains valid.

\section{GENERAL DISCUSSION}

Based on current notions regarding the function of knowledge structures and mental imagery in verbal information processing, the purpose of this study was to investigate the specific role of these factors in recognition of indeterminacy in conditional reasoning. Our aim was to clarify in theoretically meaningful terms previous content effects found in that task domain. In an initial attempt, Falmagne et al. (1985) suggested that imaginal problem representations may influence reasoning processes in verbal reasoning tasks. Results of the present research suggest that schema accessibility and mental imagery jointly were important in the reasoning process.

Why did subjects in the HR group show a relatively high level of performance on indeterminate problems? The most straightforward account of our findings, given the rationale presented above, is that clause relatedness permits the access of knowledge schemata and consequently the elaboration of problems with world knowledge. This affects reasoning by making available alternative, schemarelated situations that can then be used as counterexamples to invalid inferences. Furthermore, the relatively high performance of the HR group, and not of the LR group, indicates that imagery is necessary for this process to occur. On this account, mental imagery plays an auxiliary role in the service of elaboration (which itself plays an auxiliary role in the service of logical processing). When material permits elaboration because of relatedness of clauses, imagery is needed to facilitate representation of problem content in working memory while elaborative 
processes take place. Additionally, or alternatively, images allow better representation of added content once elaboration occurs.

On this account, subjects reasoning with high-imagery unrelated clauses (HU) generally performed poorly because they were unable to integrate content with world knowledge. Subjects reasoning with related clauses but low-imagery material (LR) also performed poorly, this time because imagery was needed to support elaborative processing. Interview data is consistent with this tentative model. In their response justifications, HR subjects frequently mentioned specific counterexamples to invalid inferences, suggesting that elaboration and use of counterexamples were important to their problem-solving procedures. Also, interestingly, images described during interviews of HR subjects were often of counterexamples rather than simply of premises given. This is consistent with the notion, proposed above, that imagery played a role in elaborative processing (as opposed to simply supporting the representation of premises in memory). Although some interviewed subjects in other groups also reported specific counterexamples, we propose that the greater number of successful subjects in the HR group is due to easier generation of counterexamples made possible by the material they received.

One possible alternative account of the results for indeterminate problems was raised earlier: imagery alone, rather than the joint effect of imagery and schema accessibility, may have led to the superior performance of $H R$ subjects, and unrelated clauses may not have enabled imagery formation. However, the relatively high imagery ratings given to high-imagery unrelated-clause conditional sentences in Experiment 2 suggest that the quasiequivalence in performance among $L U, L R$, and $H U$ subjects was not due to the low imagery value of $\mathrm{HU}$ material. Yet HU sentences were also significantly lower in imagery value than HR sentences, suggesting that the superiority of $\mathrm{HR}$ to $\mathrm{HU}$ subjects on the indetermmate problems could have been due partially to increased availability of imagery in addition to other effects of the relatedness variable.

One might also conjecture that the inferiority of the $\mathrm{HU}$ and LR groups on indeterminate problems was due only to memory factors. Specifically, premises may be difficult to hold in working memory when clauses are either unrelated or low in imagery. On this account, we would expect $H U$ and LR subjects to show relatively poor performance on determinate problems as well as indeterminate problems, particularly on those with negations, since negations increase the difficulty of representing information in working memory. However, there were no significant differences among these groups on any determinate problems. These results suggest that memory difficulties cannot account for the inferiority of the HU and LR subjects on the indeterminate problems.

Thus, for indeterminate problems, the effect of clause relatedness and imagery value does not seem to occur only at the level of initial comprehension and memory for premises. Rather, findings suggest that these factors enabled access to and representation of world knowledge that influenced the logical processing of problems.

One inconsistent result was that LU subjects were relatively successful on one indeterminate sylogism (the AC inference). Their overall performance suggests that this may be due to a conjunction of two factors. First, their impoverished problem content may have led them to approach the task differently from other subjects, particularly HR subjects, relying heavily on a formal problem representation and not using problem content. This is supported by the relatively low percentage of imagery reports among LU subjects. Also during interviews, response justifications using specific counterexamples were given in 6 out of 12 cases by HR subjects, but in only 1 out of 7 cases by LU subjects. Second, the overall response pattern of the LU group suggests that those subjects may have used a general inference schema that does not permit a definite conclusion when the second premise provides information about the consequent of the first premise. Thus, indeterminate responses are given to the AC syllogism (which affirms the consequent) and to the MT syllogism (which denies the consequent). In support of this, LU subjects performed poorly on MT relative to other groups, and almost all errors were due to "can't tell" responses."

Performance on determinate problems seems also to have been affected by the impoverished content given to the LU group, but in a different way, that is, by taxing working memory. An additional unexpected finding was the relatively low performance of $\mathrm{LU}$ subjects on determinate problems with negations in the initial premise. Although memory load did not interfere with performance of either LR or HU subjects, when problems were both low in imagery value and unrelated, memory for material may have been difficult. This possibility is supported by performance patterns for the MP and MPN syllogism. For all groups, with the exception of the LU group, performance on the affirmative and negative versions of this basic syllogism was high and equivalent. For LU subjects, however, performance on the negative was lower than on the affirmative version (errors were primarily due to "yes" responses). The negation may have interfered with accurate representation of the problem, as would be expected if the load on working memory was too great. Thus, in contrast to performance of the other groups, performance of the LU group, which was given particularly impoverished problem content, may have been affected by subjects' memory problems.

Returning to the main results, the present study enables us to refine conclusions from previous studies by identifying effective properties of meaningful material and by suggesting a process through which these properties are effective. Specifically, imagery value and clause relatedness were found jointly to affect the reasoning processes involved in judgments of indeterminacy, presumably be- 
cause (1) relatedness permitted access of knowledge schemata and schema-related elaborative processing, allowing generation of counterexamples to invalid inferences, and (2) imaginal representations supported elaboration as well as processing of problems in working memory. These two factors were found to be jointly necessary; neither factor was effective alone. Thus, previous content effects in reasoning tasks can be given a more specific interpretation that fits within accounts of the importance of prior knowledge schemata and imagery in sentence processing in general.

It is important to articulate the bearing of these results on our understanding of the relation between content factors and logical processing, especially in the light of recent proposals by Cox and Griggs (1982), Griggs and Cox (1982), and Pollard (1982).

These authors assert that when meaningful content leads to improved performance in verbal reasoning tasks, performance is exclusively based on the use of nonlogical procedures, which successfully generate a problem solution. In their view, positive content effects would only be found when material is realistic and elicits particular, episodic memories that guide subjects' responses (fortuitously consistent with correct conclusions) without facilitating insight into the logical structure of a problem. At least two objections to this view can be raised. First, although Cox and Griggs (1982) provide an extensive review of selection-task studies (Wason, 1968), suggesting that content effects are only found in that task when material is personally familiar, studies of standard conditional reasoning tasks (including the present study) show that content describing arbitrary, hypothetical situations is effective as well (e.g., Falmagne et al., 1985; Kuhn, 1977 ; Roberge \& Paulus, 1971). Reasoning problems in the present study were designed to access schemata describing possible states of affairs; they did not permit access to factual knowledge that could guarantee correct conclusions. Also, in a recent study of the selection task, material that was meaningful but not personally familiar led to improved performance (Hoch \& Tschirigi, 1983). Thus, subjects' correct responses must be accounted for by something more than availability of memories.

A second conceptual problem with the Pollard (1982) and Cox and Griggs (1982) account is related to subjects' ability to differentiate between indeterminate and determinate problems. If the use of factual knowledge, in the absence of logical understanding, is the only source of subjects' rejection of invalid inferences, then that same process could generate indeterminate responses to all logical forms with equal probability. For the problems used here, it is equally possible to generate counterexamples to valid and invalid inferences based on elaborative information. (Content was not factually biased and was crossed with logical form; thus, an antecedent could be imagined with an alternative consequent, as well as a consequent imagined with an alternative antecedent.) However, results in this and other studies show that, in general, subjects receiving content that improves perfor- mance on indeterminate problems also give correct, determinate responses to determinate problem forms.

A more plausible account of the relation between content-related knowledge and logical knowledge is that when an instance of a particular conclusion or counterexample is accessed in memory, this suggests a possible relation between connected propositions that subjects might not otherwise have considered. Subjects then evaluate this possibility in terms of its consistency with the logical relation specified by the connective. Thus, the role of content is to elicit examples subject to further logical check. Reasoning is guided by logical understanding even if content factors operate. Thus, although subjects sometimes may rely on factual knowledge alone to form conclusions, this phenomenon does not account for the general effect of world knowledge on logical processing. The results of this study suggest that accessing knowledge schemata, in conjunction with an imaginal representation of information accessed, assists in the recognition of the logical structure of conditional reasoning problems.

\section{REFERENCES}

ANDERSON, J. R., \& REDER, L. M. (1979). Levels of processing in human memory. Hillsdale, $\mathrm{NJ}$ : Erlbaum.

BLock, N. (1981). Introduction: What is the issue? In N. Block (Ed.), Imagery (pp. 1-18). Cambridge, MA: MIT Press.

BRAINE, M. D. S. (1977). On the relation between the natural logic of reasoning and standard logic. Psychological Review, 85, 1-21.

Braine, M. D. S., Reiser, B. J., \& Rumain, B. (1984). Some empirical justification for theory of natural propositional logic. In G. H. Bower (Ed.), The psychology of learning and motivation: Advances in research and theory (Vol. 18). New York: Academic Press.

Bransford, J. D., \& Franks, J. J. (1971). The abstraction of linguistic ideas. Cognitive Psychology, 2, 331-350.

Cox, J. R., \& GRIGGS, R. A. (1982). The effects of experience on performance in Wason's selection task. Memory \& Cognition, 10, 496-502.

EDDY, J. K., \& GLAss, A. L. (1981). Reading and listening to high and low imagery sentences. Journal of Verbal Learning \& Verbal Behavior, 20, 333-345

Falmagne, R. J. (1980). The development of logical competence: A psycholinguistic perspective. In R. H. Kluwe \& H. Spada (Eds.), Developmental models of thinking (pp. 171-195). New York: Academic Press.

Falmagne, R. J., Singer, J., \& Clement, C. A. (1985). Effects of imagery and temporal structures of clause on adult conditional reasoning. Unpublished manuscript.

FoDor, J. A. (1981). Imagistic representation. In N. Block (Ed.), Imagery (pp. 63-86). Cambridge, MA: MIT Press.

Griggs, R. A., \& Cox, J. R. (1982). The elusive thematic-materials effect in Wason's selection task. British Journal of Psychology, 73, 407-420.

Hoch, S. J., \& TschiRIGI, J. E. (1983). Cue redundancy and extra logical inferences in a deductive reasoning task. Memory \& Cognition, 11, 200-209.

KaUfMANN, G. (1980). Imagery, language, and cognition: Toward a theory of symbolic activity in human problem solving. Bergen, Norway: Universitetsforlaget.

Kosslyn, S. M. (1980). Image and mind. Cambridge, MA: Harvard University Press.

Kosslyn, S. M. (1981). The medium and the message in mental imagery: A theory. In N. Block (Ed.), Imagery (pp. 207-244). Cambridge, MA: MIT Press.

Kunn, D. (1977). Conditional reasoning in children. Developmental Psychology, 13, 342-353. 
Marschark, M., \& Paivio, A. (1977). Integrative processing of concrete and abstract sentences. Journal of Verbal Learning \& Verbal Behavior, 16, 217-231.

Paivio, A. (1971). Imagery and verbal processes. New York. Holt, Rinehart \& Winston.

Pollard, P. (1982). Human reasoning: Some possible effects of ava!tability. Cognition, 12, 65-96.

PyLYShyn, A. (1981). The imagery debate: Analog media versus tacit knowledge. In N. Block (Ed.), Imagery (pp. 151-206) Cambridge, MA: MIT Press.

REDER, L. M. (1980). The role of elaboration in the comprehension and retention of prose: A critical review. Review of Educational Research, 1, 5-53.

ReVLin, R., \& LeIRER, V. O (1978). The effect of personal biases on syllogistic reasoning: Rational decisions from personalized representations. In R. Revlin \& R. E. Mayer (Eds.), Human reasoning (pp. 51-81). Washington, DC: Winston.

Roberge, J. J., \& Paulus, D. H. (1971). Developmental patterns for children's class and conditional reasoning abilities. Developmental $P s y-$ chology, 4, 191-200.

RumelHart, D. E. (1980). Schemata: The building blocks of cognition. In R. Spiro, B. Bruce, \& W. Brewer (Eds.), Theoretical issues in reading comprehension (pp. 33-58). Hillsdale, NJ. Erlbaum

SCHANK, R. C. (1981). Language and memory. In D. A. Norman (Ed ), Perspectives on cognitive science (pp. 105-146). Norwood, NJ- Ablex.

Shaver, P., Pierson, L., \& Lang, S. (1974). Converging evidence for the functional significance of imagery in problem solving. $\mathrm{Cog}$ nition, 3, 359-375.

SHEPARD, R. N. (1978). The mental image. American Psychologist, 33, 125-137.

STAUDENMA YER, H. (1975). Understanding conditional reasoning with meaningful propositions. In R. J. Falmagne (Ed.), Reasoning, representation and process in children and adults (pp. 55-79). Hillsdale, NJ: Erlbaum.

WASON, P. C. (1968). Reasoning about a rule. Quarterly Journal of Experimental Psychology, 20, 273-281
WASON, P. C., \& Johnson-LAIRD, P. N. (1972). Psychology of reasoning Structure and content. Cambridge, MA: Harvard University Press.

\section{NOTES}

1 The four syllogisms are. modus ponens (MP)-If $P$ then $Q$. Given: P. Question: Q? Answer: YES; modus tollens (MT)-If P then Q. Given Not Q. Question. P? Answer: NO, denying the antecedent (DA)-If $P$ then Q. Given. Not P. Question: Q? Answer: CAN'T TELL; affirming the consequent ( $\mathrm{AC}$ )-If $P$ then $\mathrm{Q}$. Given: $\mathrm{Q}$. Question: P? Answer: CAN'T TELL. A meaningful example of the last syllogism is: "If the man wears a green shirt, then he wears plaid pants. The man wears plaid pants. Does he wear a green shirt?" CAN'T TELL.

2 . There were no significant differences among the groups on the number of determinate or indeterminate pretest problems correct. For ex ample, for indeterminate problems, the means and standard deviations for the HR, HU, LR, and LU groups, respectively, were 4.15, 1.31; $3.95,1.31 ; 3.75,1.21 ; 4.10,1.37$

3. Recall that each content group was divided into subgroups, each receiving a different pairing of sentence subsets with logical form. In each content group, for the indeterminate inferences taken together, the difference between subgroups was greater than the difference between sentence subsets, indicatıng that subgroup variability was a product of subject differences rather than sentence differences. Therefore, in testing for differences between content groups, we collapsed over subgroups, treatıng subgroup variability as subject variability.

4. This response pattern is consistent with Braine's (1977) hypothesis that the natural language representation of "if $p$ then $q$ " is a directional inference schema which moves the reasoner from information about $p$ to information about $q$, and provides no conclusion when information is given about $q$. Without a reductio ad absurdum line of reasoning, then, subjects will often give indeterminate responses to modus tollens.

(Manuscript received June 24, 1985; revision accepted for publication December 30, 1985.) 